\title{
Dynamics of the star S0-1 and the nature of the compact dark object at the Galactic center
}

\author{
F. Munyaneza, D. Tsiklauri", and R.D. Viollier \\ Physics Department, University of Cape Town, Rondebosch 7701, South Africa
}

\begin{abstract}
It has been recently shown (Munyaneza, Tsiklauri and Viollier 1998) that the analysis of the orbits of the fast moving stars close to Sgr A* (Eckart and Genzel 1997) provides a valuable tool to probe the gravitational potential near the Galactic center. As an example, we present here the results on a calculation of possible orbits of the star S0-1 in both, the black hole and degenerate neutrino ball scenarios of the central mass, based on the recent measurements of stellar proper motions at the Galactic center by Ghez et al. 1998. Taking into account the error bars of their analysis, it is shown that within a few years time, the orbit of S0-1 may indeed reveal the nature of the supermassive compact dark object at the Galactic center.
\end{abstract}

Subject headings: black hole physics — celestial mechanics, stellar dynamics — dark matter elementary particles - Galaxy: center

\section{Introduction}

The determination of the mass distribution near the center of our Galaxy and the question, whether it harbours a supermassive black hole (BH) or not, have been long-standing issues (Oort 1977; Genzel and Townes 1987; Genzel et al. 1994 and Ho 1998 for a recent review). Various techniques have been used to find the mass of this supermassive compact dark object which is usually identified with the radio source Sagittarius A* (Sgr A*) at or near the Galactic center. The most detailed information to date comes from the statistical analysis of the dynamics of stars moving in the gravitational field of the central mass distribution (Sellgren et al. 1987; Rieke and Rieke 1988; McGinn et al. 1989; Sellgren et al. 1990; Lindqvist et al. 1992; Haller et al. 1996; Eckart and Genzel 1996; Genzel et al. 1996; Eckart and Genzel 1997; Genzel et al. 1997; Ghez et al. 1998). Genzel et al. 1997 have established that the central dark object has a mass of $(2.61 \pm 0.76) \times 10^{6} M_{\odot}$, concentrated within a radius of $0.016 \mathrm{pc}$ and located very close to Sgr A*. In the most recent observations, Ghez at al. 1998 confirm a mass of $(2.6 \pm 0.2) \times 10^{6} M_{\odot}$, enclosed within a radius of $0.015 \mathrm{pc}$. In the latter observations, the accuracy of the velocity measurements in the central $\operatorname{arcsec}^{2}$ has been improved considerably, and thus the error bar on the central mass has been reduced by about a factor of 4 . In both data sets, the presence of a supermassive compact dark object is revealed by the fact that several stars are moving within a projected distance of less than $0.01 \mathrm{pc}$ from the central radio source Sgr $\mathrm{A}^{*}$ at projected velocities in excess of $1000 \mathrm{~km} / \mathrm{s}$.

For completeness, we mention here that the mass distribution at the Galactic center could also be studied through the motion of gas clouds and streamers (Lacy et al. 1980; Genzel \& Townes 1987; Lacy et al. 1991). However, gas flows may be easily perturbed by non-gravitational forces such as shocks, radiation

\footnotetext{
${ }^{1}$ permanent address: Physics Department, Tbilisi State University, 3 Chavchavadze Ave., 380028 Tbilisi, Georgia
} 
pressure, winds, magnetic fields,etc., and hence this probe is considered to be less reliable for determining the mass of the compact dark object at the Galactic center.

The non-thermal spectrum of Sgr A* (Serabyn et al. 1997), that has been shown to originate from a very compact source (Rogers et al. 1994; Genzel et al. 1997; Ghez et al. 1998), and the low proper motion of Sgr A* (Backer 1996) have led many (e.g. Lynden-Bell and Rees 1971) to suggest that Sgr A* may be a supermassive BH of mass $\sim 2.6 \times 10^{6} M_{\odot}$. Supermassive BHs have also been inferred for several other galaxies such as M87 (Ford et al. 1994; Harms et al. 1994; Macchetto et al. 1997) and NGC 4258 (Greenhill et al. 1995; Myoshi et al. 1995). Taking this suggestion seriously, one is immediately faced with fundamental issues such as the prevalence of supermassive BHs in the nuclei of normal galaxies and the nature of the accretion mechanism that makes Sgr A* so much fainter than typical active galactic nuclei (Melia 1994; Narayan et al. 1995). However, as the best current observations probe the gravitational potential at radii $4 \times 10^{4}$ larger than the Schwarzschild radius of a $\mathrm{BH}$ of mass $2.6 \times 10^{6} M_{\odot}$ (Ghez et al. 1998), it is perhaps prudent not to focus too much on the BH scenario, without having explored alternative scenarios for the supermassive compact dark object.

One alternative to the BH scenario is a very compact stellar cluster (Haller et al. 1996, Sanders, 1992). However, based on the evaporation and collision time stability criteria, it is doubtful that such clusters could have survived up to the present time (see Moffat 1997 for an alternative point of view). Indeed, in the case of our Galaxy and NGC 4258, Maoz $(1995,1998)$ has found that even the lower limits to the half-mass densities of such compact clusters $\left(1 \times 10^{12} M_{\odot} \mathrm{pc}^{-3}\right.$ for NGC 4258 and $6 \times 10^{11} M_{\odot} \mathrm{pc}^{-3}$ for our Galaxy) are too large that they could be due to stable clusters of stellar or substellar remnants. The estimated maximal lifetimes for such dense clusters are about $10^{8}$ years for our Galaxy and a few $10^{8}$ years for the NGC 4258, i.e. much shorter than the age of the Universe. This seems to rule out the existence of dense clusters at the centers of the above mentioned galaxies, unless we are prepared to believe that we happen to live in a privileged epoch of the lifetime of the Universe. Note, however, that for other galaxies, such as M31, M32, M87, NGC 3115, NGC 3377, NGC 4261, NGC 4342, NGC 4486B and NGC 4594, maximal lifetimes of dense stellar clusters are in excess of $10^{11}$ years. Moreover, it should be acknowledged that the uncertainties in the understanding of the core collapse process of such dense clusters still leave some room for speculation about a possible interpretation of the supermassive compact dark objects at the centers of galaxies (including both, our Galaxy and NGC 4258) in terms of e.g. core-collapsed clusters (Maoz 1998). But, apart from a cluster of very low mass BH's that is free of stability problems, the most attractive alternative to a dense stellar cluster is a cluster of elementary particles.

In fact, in the recent past, an alternative model for the supermassive compact dark objects in galactic centers has been developed (Viollier et al. 1992, 1993; Viollier 1994; Tsiklauri and Viollier 1996, 1998a,b, 1999; Bilić et al. 1998; Bilić et al. 1999). The cornerstone of this model is that the dark matter at the center of galaxies is made of nonbaryonic matter in the form of massive neutrinos that interact gravitationally forming supermassive neutrino balls in which the degeneracy pressure of the neutrinos balances their self-gravity. Such neutrino balls could have been formed in the early Universe during a first-order gravitational phase transition (Bilić and Viollier 1997,1998,1999a,b). In fact, it has been recently shown that the dark matter concentration observed through stellar motion at the Galactic center (Eckart \& Genzel 1997; Genzel et al. 1996) is consistent with a supermassive object of $2.5 \times 10^{6}$ solar masses made of self-gravitating, degenerate heavy neutrino matter (Tsiklauri \& Viollier 1998a). Moreover, it has been shown that an acceptable fit to the infrared and radio spectrum above $20 \mathrm{GHz}$, which is presumably emitted by the compact dark object, can be reproduced in the framework of standard accretion disk theory (Tsiklauri \& Viollier 1999; Bilić et al. 1998), in terms of a baryonic disk immersed in the shallow potential 
of the degenerate neutrino ball of $2.5 \times 10^{6}$ solar masses.

The purpose of this paper is to compare the predictions of these two models for the supermassive compact dark object at the center our Galaxy, i.e. (i) the black hole scenario and (ii) the degenerate neutrino ball scenario as an example of an extended object. Both these models are not in contradiction with the technologically challenging proper motions observations and their statistical interpretation (Genzel et al. 1997; Ghez et al. 1998) . It is therefore desirable to have an additional independent dynamical test, in order to distinguish between these two possible scenarios describing the compact dark object at the center of our Galaxy. In the recent past, mainly statistical arguments involving many stars have been used to determine the gravitational potential at the Galactic center. However, in this paper, we would like to demonstrate that it is also possible to draw definite conclusions from the motion of individual stars, in particular, in the immediate vicinity of the Galactic center, where statistical arguments cannot be easily applied due to the low density of stars. To this end, we have recently calculated the orbits (Munyaneza, Tsiklauri and Viollier 1998) of the fastest moving infrared source S1 using the Genzel et al. 1997 data for a supermassive $\mathrm{BH}$ or a neutrino ball mass of $2.61 \times 10^{6}$ solar masses. We have shown that tracking the orbits of S1 offers a good opportunity to distinguish in a few years time between the two scenarios for the supermassive compact dark object. Here we perform a full analysis of the orbits of the same star S0-1 based on the most recent Ghez et al. 1998 data, including all the error bars of the measurements. A distance to the Galactic center of $8 \mathrm{kpc}$ has been assumed throughout this paper.

This paper is organized as follows: In section 2, we present the equations that describe degenerate neutrino balls and we establish some constraints on the neutrino mass based on Ghez et al. 1998 data. In section 3, we study the dynamics of S0-1 and conclude with the discussion in section 4 .

\section{The compact dark object as a neutrino ball}

Dark matter at the Galactic center can be described by the gravitational potential $\Phi(r)$ of the neutrinos and antineutrinos that satisfies Poisson's equation

$$
\Delta \Phi=4 \pi G \rho_{\nu},
$$

where $G$ is Newton's gravitational constant and $\rho_{\nu}$ is the mass density of the neutrinos and antineutrinos. Neutrino matter will interact gravitationally to form supermassive neutrino balls in which self-gravity of the neutrinos is being balanced by their degeneracy pressure $P_{\nu}(r)$ according to the equation of hydrostatic equilibrium

$$
\frac{d P_{\nu}}{d r}=-\rho_{\nu} \frac{d \Phi}{d r}
$$

In order to solve equation (1) , one needs a relation between the pressure $P_{\nu}$ and the density $\rho_{\nu}$. To this end we choose the polytropic equation of state of degenerate neutrino matter, i.e.

$$
P_{\nu}=K \rho_{\nu}^{5 / 3}
$$

where the polytropic constant $K$ is given by (Viollier, 1994)

$$
K=\left(\frac{6}{g_{\nu}}\right)^{2 / 3} \frac{\pi^{4 / 3} \hbar^{2}}{5 m_{\nu}^{8 / 3}} .
$$

Here, $m_{\nu}$ denotes the neutrino mass, $g_{\nu}$ is the spin degeneracy factor of the neutrinos and antineutrinos, i.e. $g_{\nu}=2$ for Majorana and $g_{\nu}=4$ for Dirac neutrinos and antineutrinos. We now introduce the dimensionless 
potential and radial variable, $v$ and $x$, by

$$
\begin{gathered}
\Phi(r)=\frac{G M_{\odot}}{a_{\nu}}\left(v^{\prime}\left(x_{0}\right)-\frac{v(x)}{x}\right), \\
r=a_{\nu} x,
\end{gathered}
$$

where $x_{0}$ is the dimensionless radius of the neutrino ball, and the scale factor $a_{\nu}$ which plays here the role of a length unit is given by

$$
a_{\nu}=2.1376 \mathrm{lyr} \times\left(\frac{17.2 \mathrm{keV}}{m_{\nu} c^{2}}\right)^{8 / 3} g_{\nu}^{-2 / 3} .
$$

Assuming spherical symmetry, we finally arrive at the non-linear Lané-Emden equation

$$
\frac{d^{2} v}{d x^{2}}=-\frac{v^{3 / 2}}{x^{1 / 2}}
$$

with polytropic index $3 / 2$. The boundary conditions are chosen in such a way that $v$ vanishes at the boundary $x_{0}$ of the neutrino ball. The mass $M_{B}$ of a (pointlike) baryonic star at the center of the neutrino ball is fixed by $v(0)=M_{B} / M_{\odot}$. The case $M_{B}=0$ corresponds to a pure neutrino ball without a pointlike

source at the center. The mass enclosed within a radius $r$ in a pure neutrino ball can be written in terms of $v(x)$ and its derivative $v^{\prime}(x)$ as

$$
M(r)=\int_{0}^{r} 4 \pi \rho_{\nu} r^{2} d r=-M_{\odot}\left(v^{\prime}(x) x-v(x)\right) .
$$

In order to describe the compact dark object at the Galactic center as a neutrino ball and constrain its physical parameters appropriately, it is worthwhile to use the most recent observational data by Ghez et al. 1998, who established that the mass enclosed within $0.015 \mathrm{pc}$ at the Galactic center is $(2.6 \pm 0.2) \times 10^{6}$ solar masses. Following the analysis of Tsiklauri \& Viollier 1998a, we choose the minimal neutrino mass $m_{\nu}$ to reproduce the observed matter distribution, as can be seen from Fig. 1, where we have added the Ghez et al. 1998 and Genzel et al. 1997 data points with error bars. In Fig. 1 we include only the neutrino ball contribution to the enclosed mass, as the stellar cluster contribution is negligible by orders of magnitude at these radii. For a $M=2.4 \times 10^{6} M_{\odot}$ neutrino ball, the constraints on the neutrino mass are $m_{\nu} \geq 17.50 \mathrm{keV} / c^{2}$ for $g_{\nu}=2$ and $m_{\nu} \geq 14.72 \mathrm{keV} / c^{2}$ for $g_{\nu}=4$, and the radius of the neutrino ball is $R \leq 1.50 \times 10^{-2} \mathrm{pc}$. Using the value of $M=2.6 \times 10^{6} M_{\odot}$, the bounds on the neutrino mass are $m_{\nu} \geq 15.92 \mathrm{keV} / c^{2}$ for $g_{\nu}=2$ or $m_{\nu} \geq 13.39 \mathrm{keV} / c^{2}$ for $g_{\nu}=4$ and the radius of the neutrino ball turns out to be $R \leq 1.88 \times 10^{-2} \mathrm{pc}$. Finally, for a $M=2.8 \times 10^{6} M_{\odot}$ neutrino ball, the range of neutrino mass is $m_{\nu} \geq 15.31 \mathrm{keV} / c^{2}$ for $g_{\nu}=2$ and $m_{\nu} \geq 12.87 \mathrm{keV} / c^{2}$ for $g_{\nu}=4$ and the corresponding neutrino ball radius $R \leq 2.04 \times 10^{-2} \mathrm{pc}$.

\section{Dynamics of S0-1}

We investigate the motion of S0-1 that is the star closest to the Galactic center, and at the same time, also the fastest of the 15 stars in the central $\operatorname{arcsec}^{2}$ around Sgr A*. We study the motion of S0-1 in the gravitational potential near Sgr $\mathrm{A}^{*}$, assuming that the central object is either a $\mathrm{BH}$ of mass $M$ or a spatially extended object represented by a neutrino ball of mass $M$, that consists of self-gravitating degenerate heavy neutrino matter. The $\mathrm{BH}$ or neutrino ball mass $M$ will be taken to be 2.4, 2.6 and 2.8 $\times 10^{6}$ solar masses which corresponds to the range allowed by the Ghez et al. 1998 data. We use Newtonian 
dynamics, as the problem is essentially nonrelativistic, because the mass of the neutrino ball is much less than the Oppenheimer-Volkoff limit corresponding to this particular neutrino mass (Bilić, Munyaneza \& Viollier 1999). Consequently, we can write Newton's equations of motion as

$$
\begin{aligned}
& \ddot{x}=-\frac{G M(r)}{\left(x^{2}+y^{2}+z^{2}\right)^{3 / 2}} x, \\
& \ddot{y}=-\frac{G M(r)}{\left(x^{2}+y^{2}+z^{2}\right)^{3 / 2}} y, \\
& \ddot{z}=-\frac{G M(r)}{\left(x^{2}+y^{2}+z^{2}\right)^{3 / 2}} z,
\end{aligned}
$$

where $x, y, z$ denote the components of the radius vector of the star S0-1 and $r=\sqrt{x^{2}+y^{2}+z^{2}}$, Sgr A* being the origin of the coordinate system. We thus assume that the center of the neutrino ball and the $\mathrm{BH}$ is at the position of Sgr A*. The dot denotes of course the derivative with respect to time. In the case of a BH, $M(r)=M$ is independent of $r$, while in the neutrino ball scenario, $M(r)$ is given by Eq. (9) and it reaches $M(R)=M$ at the radius of the neutrino ball $R$. The initial positions and velocities for this system of equations are taken to be those of S0-1 in 1995.4, when the coordinates of S0-1 were RA $=-0.107^{\prime \prime}$ and DEC $=0.039^{\prime \prime}$. The $x$ and $y$ components of the projected velocity are $v_{x}=470 \pm 130 \mathrm{~km} / \mathrm{s}$ and $v_{y}=-1330 \pm 140 \mathrm{~km} / \mathrm{s}$ (Ghez et al. 1998), respectively. Here $x$ is opposite to the RA direction and $y$ is in the DEC direction.

In Fig. 2 we plot two typical orbits of S0-1 corresponding to a BH and neutrino ball mass of $M=2.6 \times 10^{6} M_{\odot}$. The input values for $v_{x}$ and $v_{y}$ are $470 \mathrm{~km} / \mathrm{s}$ and $-1330 \mathrm{~km} / \mathrm{s}$, respectively. The $z$-coordinate of the star S0-1 is assumed to be zero and the velocity component in the line-of-sight of the star S0-1, $v_{z}$, has also been set equal to zero in this graph. The filled square labels denote the time in years from 1990 till 2015. In the case of a BH, the orbit of S0-1 is an ellipse, with Sgr A* being located in one focus (denoted by the star in the figure). The period of $\mathrm{S} 0-1$ is 12.7 years and the minimal and maximal distances from Sgr $\mathrm{A}^{*}$ are 1.49 and 7.18 light days, respectively. In the case of a neutrino ball, the orbit will be bound but not closed, with minimal and maximal distances from Sgr A* of 3.98 and 42.07 light days, respectively. It can be seen from Fig. 2 that, in the case of a neutrino ball, S0-1 is deflected much less than for a $\mathrm{BH}$, as the gravitational force at a given distance from $\mathrm{Sgr} \mathrm{A}^{*}$ is determined by the mass enclosed within this distance. Using Eq. (9) we can estimate the mass enclosed within a radius corresponding to the projected distance of S0-1 from Sgr A* $\left(4.41 \times 10^{-3} \mathrm{pc}\right)$ to be $\sim 1.8 \times 10^{5} M_{\odot}$. Thus, in the case of a neutrino ball, the force acting on S0-1 is about 14 times less than in the case of a BH. This graph can serve to establish, whether Sgr $\mathrm{A}^{*}$ is a $\mathrm{BH}$ or an extended object, due to the fact that the positions of S0-1 will differ as time goes on in the two scenarios. However, this conclusion is perhaps too optimistic, as we have not yet considered (i) the uncertainties in $v_{x}$ and $v_{y}$, (ii) the error bars in the total mass of the $\mathrm{BH}$ or neutrino ball, (iii) the complete lack of information on $z$ and $v_{z}$.

As a next step, we investigate the dependence of the orbits on the uncertainties in the velocity components. The results of this calculation are presented in Fig. 3 where we have set $z=v_{z}=0$. In the case of a BH, the orbits of S0-1 are ellipses, while the other 5 thick lines are bound orbits of S0-1 for the neutrino ball scenario. The spread of the orbits induced by the error bars in $v_{x}$ and $v_{y}$ is small compared to that of the recent analysis based on the Genzel et al. 1997 data (Munyaneza, Tsiklauri \& Viollier 1998). The time labels, represented by filled squares on the orbits, are placed in intervals of 5 years: starting from 1995.4 up to 2005 in the case of a BH, and up to 2015 in the case of a neutrino ball. The periods of S0-1 for different orbits vary between 10 and 17 years for the BH scenario. We thus see that the error bars in $v_{x}$ 
and $v_{y}$ do not alter the predictions of Fig. 1 in substance. We now would like to study, how the orbits are changed if we let the mass of the neutrino ball or the $\mathrm{BH}$ vary within the estimated error bars (Ghez et al. 1998).

In Fig. 4 and 5, we present the results of our calculations, for both scenarios, with central masses of $M=2.4 \times 10^{6} M_{\odot}$ and $M=2.8 \times 10^{6} M_{\odot}$, respectively. The neutrino masses consistent with the Ghez et al. (1998) data are are $m_{\nu} \geq 17.50 \mathrm{keV} / c^{2}$ for a $M=2.4 \times 10^{6} M_{\odot}$ neutrino ball and $m_{\nu} \geq 15.31 \mathrm{keV} / c^{2}$ for a $M=2.8 \times 10^{6} M_{\odot}$ neutrino ball. The filled squares represent the time labels spaced by 5 year intervals as in Fig. 3. In the BH scenario, the periods of S0-1 with $M=2.4 \times 10^{6} M_{\odot}$ vary between 11 years and 20 years, while in the case of a $M=2.8 \times 10^{6} M_{\odot}$, the periods vary between 9.5 and 15 years. Comparing the orbits of S0-1 in Fig. 4 and 5 with those in Fig. 3, we conclude that the errors bars in the total mass of the BH or neutrino ball make no qualitative difference for the motion of S0-1. In both scenarios of the supermassive compact dark object, all the orbits considered for three different values of the $\mathrm{BH}$ or neutrino ball mass are bound for $z=v_{z}=0$, as can be seen from Fig. 6 and 7, where the escape and circular velocities are plotted as functions of the distance from Sgr $\mathrm{A}^{*}$. In these graphs, we have also included the Ghez et al. (1998) data with error bars, for the 15 stars in the central $\operatorname{arsec}^{2}$, assuming that the velocity component and distance from Sgr $\mathrm{A}^{*}$ in the line-of-sight are both zero, i.e. $v_{z}=0$ and $z=0$. Thus, the data points are lower bounds on the true circular or escape velocity and radius, and the real values lie in the quarter-plane to the right-and-up of the measured data point. For instance, the innermost data point describing the star S0-1 is in both scenarios, consistent with a bound orbit if $|z|$ and $\left|v_{z}\right|$ are not too large, as can be seen from the escape velocity in Fig. 6. However, S0-1 cannot be interpreted as a virialized star in the neutrino ball scenario, as is evident from the plot of the circular velocity in Fig 7 ; it thus would have to be an intruder star. If the projected velocity of a star at a given projected distance from Sgr $\mathrm{A}^{*}$ is larger than the escape velocity at the same distance (assuming $z=0$ ), the neutrino ball scenario is virtually ruled out, since the kinetic energy of the star would have to be very large at infinity.

We now turn to the investigation of the dependence of the orbits on the $z$ coordinate and $z$ component of the velocity of the star S0-1. The two quantities, $z$ and $v_{z}$, are the major source of uncertainty in determining the exact orbit of the star S0-1. However, this shortcoming will not substantially affect the predictive power of our model, as we will see below. In Fig. 8 we show the results of a calculation of the dependence of the orbit on $z$ for a $M=2.6 \times 10^{6} M_{\odot}$ neutrino ball or BH. The input values for $v_{x}$ and $v_{y}$ are fixed at $470 \mathrm{~km} / \mathrm{s}$ and $-1330 \mathrm{~km} / \mathrm{s}$, respectively, and $v_{z}$ is assumed to be zero. The $z$-coordinate is varied from zero up to the radius of the neutrino ball, i.e. the distance from Sgr $\mathrm{A}^{*}$ beyond which there is obviously no difference between the $\mathrm{BH}$ and the neutrino ball scenarios. In this case, the radius of the neutrino ball $1.88 \times 10^{-2} \mathrm{pc}$ or $0.485^{\prime \prime}$. The top panel represents the orbits in the case of a BH, for different values of $z$, while the lower panel describes the dependence of the orbit on $z$ in the neutrino ball scenario. We conclude from this plot that, increasing $|z|$ has the effect of shifting the orbits towards the lower right corner of the graph. This is, obviously, due to the fact that increasing $|z|$ means going further away from the scattering center, thus yielding less deflection of the orbit. Moreover, in the neutrino ball scenario, the dependence on $z$ is relatively insignificant, as long as $|z|$ is smaller than the radius of the neutrino ball. This is in accordance with the fact that for small distances from the center, the potential of a neutrino ball can be approximated by a harmonic oscillator-type potential, where the Newtonian equations of motion decouple in Cartesian coordinates. The dependence of the orbits of S0-1 on $v_{z}$ has a similar effect as in the previous graph.Here, we have fixed $z$ to zero and $v_{z}$ has been varied as an input parameter. Increasing $\left|v_{z}\right|$ yields a greater velocity of the star and, obviously, a fast moving star will be deflected less than a star with smaller $\left|v_{z}\right|$. The results of this calculation are summarized in Fig. 9. 


\section{Conclusion and discussion}

We have demonstrated that the orbits of S0-1 differ substantially for the BH and neutrino ball scenarios of the Galactic center, especially with the new Ghez et al. (1998) data. We have shown that using these data, the error bars in velocities of S0-1 and mass of the central object do not change the pattern of the orbits of S0-1. In the case of a BH, the orbit of S0-1 is much more curved than in the neutrino ball scenario, as long as $|z|$ is smaller than the radius of the neutrino ball. Increasing $|z|$ and $\left|v_{z}\right|$ shifts the orbits to the lower right corner of the graph, and this gives us a key to establish the allowed regions of S0-1 depending on whether it is a $\mathrm{BH}$ or a neutrino ball, irrespective of the values of the parameters $z$ and $v_{z}$. In Fig. 10 three orbits are plotted: the upper-leftmost orbit of S0-1 corresponding to the neutrino ball scenario (actually, line 9 of Fig. 4) and two orbits in a BH scenario with the smallest minimal and maximal distances from Sgr $\mathrm{A}^{*}$ (ellipses 2 and 4 from Fig.5). This figure serves as a test to distinguish the supermassive BH scenario from the neutrino ball model of the Galactic center. It is clear that, as the observations proceed within the next year, one might be able to tell the difference between the two models of the supermassive compact dark object at the center of our Galaxy.

If the star is found in the region $F$ inside the ellipses, this will rule out both the $\mathrm{BH}$ and the neutrino ball scenario of Sgr $\mathrm{A}^{*}$, as seen in Fig. 10. We can estimate the minimal distance of approach to Sgr $\mathrm{A}^{*}$ to be 0.909 light days. If the orbit of S0-1 ends up in the upper-left zone of the thick line, this will clearly rule out the neutrino ball scenario for the chosen lower limit of the neutrino mass. However, if S0-1 is found in the lower right corner of the same line (i.e. below the thick line), then the supermassive object can be interpreted as either a neutrino ball or a $\mathrm{BH}$ with a large $z$ or $v_{z}$ parameter. One can of course repeat this analysis for several stars in the central $\operatorname{arcsec}^{2}$ and use some statistical arguments: should there be no stars in the black hole zone, and many stars found in the zone for black holes and neutrino balls, the black hole interpretation of the supermassive compact dark object at the Galactic center would become less attractive, as some of the stars should be moving close to the plane perpendicular to the line-of-sight, i.e. they should have small $\left|v_{z}\right|$ and $|z|$.

The neutrino masses used for the neutrino ball are lower limits. We note that increasing the neutrino

mass will make the radius smaller (the neutrino ball radius scales as $\propto m_{\nu}^{-8 / 3}$ ) and, when it reaches the mass corresponding to the Oppenheimer-Volkoff limit, there will be little difference between the two scenarios.

\section{Acknowledgements}

One of us (F. Munyaneza) gratefully acknowledges funding from the Deutscher Akademischer Austauschdienst and the University of Cape Town. This work is supported by the Foundation for Fundamental Research (FFR).

\section{REFERENCES}

Backer, D.C. 1996, in Unsolved problems in the Milky Way, eds. L. Blitz and P. Teuben, Proc. of IAU Symp. No. 169 (Dordrecht: Kluwer)

Bilić, N., Munyaneza, F., and Viollier, R.D. 1999, Phys. Rev. D59, 024003

Bilić, N., Tsiklauri, D. and Viollier, R.D. 1998, Prog. Part. Nucl. Phys. 40, 17

Bilić, N. and Viollier, R.D. 1997, Phys. Lett. B 408,75 
Bilić, N. and Viollier, R.D. 1998, Nucl. Phys. (Proc. Suppl.) B66,256

Bilić, N. and Viollier, R.D. 1999a, Gen. Rel. Grav. (to be published), gr-qc/9706019

Bilić, N. and Viollier, R.D. 1999b, Phys. Rev. D (submitted), hep-ph/9809563

Eckart, A. and Genzel, R. 1996, Nature, 383,415

Eckart, A. and Genzel, R. 1997, MNRAS, 284, 576

Ford, H.C., Harms, R.J., Tsvetanov, Z.I., Hartig, G.F., Dressel, L.L., Kriss, G.A., Bohlin, R.C., Davidsen, A.F., Margon, B., and Kochhar, A.K. 1994, ApJ, 435, L27

Genzel, R., Eckart, A., Ott, T. and Eisenhauer, F. 1997, MNRAS, 291, 219

Genzel, R., Hollenbach, D.J., and Townes, C.H. 1994, Rep. Prog. Phys. 57, 417

Genzel, R., Thatte, N., Krabbe, A., Kroker, H., and Tacconi-Garman, L.E. 1996, ApJ, 472, 153

Genzel, R. and Townes, C.H. 1987, ARA\&A, 25, 377

Ghez, A.M., Klein, B.L., Morris, M. and Becklin, E.E. 1998, ApJ, 509, 678

Greenhill, L.J., Jiang, D.R., Moran, J.M., Reid, M.J., Lo, K.Y., Claussen, M.J. 1995, ApJ, 440, 619

Haller, J. W., Rieke, M. J., Rieke, G.H., Tamblyn, P. , Close , L., and Melia , F. 1996, ApJ, 456, 194

Harms, R. J., Ford, H. C., Tsvetanov, Z.I., Hartig, G. F., Dressel, L. L., Kriss, G. A., Bohlim, R., Davidsen, A.F., Margon, B., and Kochhar, A. K. 1994, ApJ 435, L35

Ho, L. C. 1998, Invited review paper to appear in Observational Evidence for Black Holes in the Universe, ed. S.K. Chakrabararti (Dordrecht: Kluwer), astro-ph/9803307

Lacy, J.H. , Achtermann, J.M., and Serabyn, E. 1991, ApJ,380, L71

Lacy, J.H., Townes, C.H. , and Hollenbach, D.J. 1980, ApJ, 241,132

Lindqvist, M., Habing, H.J. and Winnberg, A. 1992, A\&A, 259,118

Lynden-Bell, D. and Rees, M. 1971, MNRAS, 152,461

Macchetto et al. ApJ 1997, 489, 579

Maoz, E. 1995, ApJ, 447, L91

Maoz, E. 1998, ApJ, 494, L181

McGinn,J. T., Sellgren, K., Becklin, E.E. and Hall, D.N.B. 1989, ApJ, 338,824

Melia, F. 1994, ApJ, 426, 577

Moffat, J.W. 1997, astro-ph/9704232.

Munyaneza, F. , Tsiklauri, D., and Viollier, R. D. 1998, ApJ, 509, L105

Myoshi, M., Moran, J. M., Hernstein, J., Greenhill, L., Nakai, N., Diamond, P., and Inoue, M. 1995, Nature 373,127

Narayan, R.,Yi, I., and Mahadevan, R. 1995, Nature, 374,623

Rieke, G.H. and Rieke, M.J. 1988, ApJ, 330, L33

Rogers, A.E.E., Doeleman, S., Wright, M.C.H., Bower, G.C., Backer, D.C., Padin, S., Philips, J.A., Emerson, D.T. , Greenhill., L., Moran, J.M., and Kellermann, K.I. 1994, ApJ, 434, L59

Oort, J. 1977, ARA\&A, 15, 295

Sanders, R.H. 1992, Nature, 359, 131 
Sellgren, K., Hall , D.N.B. , Kleinmann, S.G., and Scoville, N.Z. 1987, ApJ, 317, 881

Sellgren, K., McGinn, M.T., Becklin, E.E., and Hall, D.N.B. 1990, ApJ, 359, 112

Serabyn, E., Carlstrom, J., Lay, O., Lis, D.C., Hunter, T.R., and Lacy, J.H. 1997, ApJ, 490, L77

Tsiklauri, D. and Viollier, R.D. 1996, MNRAS, 282,1299

Tsiklauri, D. and Viollier, R.D. 1998a, ApJ, 500, 591

Tsiklauri, D. and Viollier, R.D. 1998b, ApJ, 501, 486

Tsiklauri, D. and Viollier, R.D. 1999, Astroparticle Phys. (submitted), astro-ph/9805272

Viollier, R.D., Leimgruber, F.R., and Trautmann, D. 1992, Phys. Lett., B297, 132

Viollier, R.D., Trautmann, D., and Tupper, G.B. , 1993, Phys. Lett., B306, 79

Viollier, R.D., 1994, Prog. Part. Nucl. Phys., 32, 51

This preprint was prepared with the AAS $\mathrm{LA}_{\mathrm{E}} \mathrm{X}$ macros v4.0. 
Figure captions:

Fig. 1: The mass enclosed within a distance of the center of a neutrino ball of 2.4, 2.6, 2.61 and 2.8 millions solar masses. Based on the Ghez et al. 1998 data, the bounds on the neutrino mass are $m_{\nu} \geq 17.50$ $\mathrm{keV} / c^{2}$ for $g_{\nu}=2$ or $m_{\nu} \geq 14.72 \mathrm{keV} / c^{2}$ for $g_{\nu}=4$ and $M=2.4 \times 10^{6} M_{\odot}$. For $M=2.6 \times 10^{6} M_{\odot}$ the bounds on the neutrino mass are $m_{\nu} \geq 15.92 \mathrm{keV} / c^{2}$ for $g_{\nu}=2$ and $m_{\nu} \geq 13.39 \mathrm{keV} / c^{2}$ for $g_{\nu}=4$. Finally, a neutrino mass range of $m_{\nu} \geq 15.31 \mathrm{keV} / c^{2}$ for $g_{\nu}=2$ or $m_{\nu} \geq 12.87 \mathrm{keV} / c^{2}$ for $g_{\nu}=4$ is consistent with a supermassive object of $M=2.8 \times 10^{6} M_{\odot}$. The Ghez et al. 1998 and Genzel et al. 1997 data points with error bars are also shown in this graph.

Fig.2: Projected orbits of the star S0-1 for BH and neutrino ball scenarios with $M=2.6 \times 10^{6} M_{\odot}$ and $v_{z}=z=0$. The velocity components of S0-1 are taken to be $v_{x}=470 \mathrm{~km} / \mathrm{s}$ and $v_{y}=-1330 \mathrm{~km} / \mathrm{s}$. The filled squares denote the time labels. The period of S0-1 in a the BH scenario is 12.7 years and the minimal and maximal distances from Sgr $\mathrm{A}^{*}$ are 1.49 and 7.18 light days. The orbit of S0-1 in the neutrino ball scenario is bound with minimal and maximal distances from Sgr A* of 3.98 and 42.07 light-days, respectively.

Fig.3: Projected orbits of the star S0-1 in the case of a BH or a neutrino ball of $M=2.6 \times 10^{6} M_{\odot}$ taking into account the error bars in the velocity components. The labels for the different orbits are: 1 : $v_{x}=470 \mathrm{~km} / \mathrm{s}$ and $v_{y}=-1330 \mathrm{~km} / \mathrm{s}$ (median values), $2: v_{x}=340 \mathrm{~km} / \mathrm{s}$ and $v_{y}=-1190 \mathrm{~km} / \mathrm{s}, 3: v_{x}=340$ $\mathrm{km} / \mathrm{s}$ and $v_{y}=-1470 \mathrm{~km} / \mathrm{s}, 4: v_{x}=600 \mathrm{~km} / \mathrm{s}$ and $v_{y}=-1190 \mathrm{~km} / \mathrm{s}, 5: v_{x}=600 \mathrm{~km} / \mathrm{s}$ and $v_{y}=-1470$ $\mathrm{km} / \mathrm{s}$. The periods of S0-1 for different orbits in the BH scenario vary between 10 and 17 years. The thick lines 6 to 10 correspond to the orbits in the neutrino ball scenario with the following description: 6 : $v_{x}=470 \mathrm{~km} / \mathrm{s}$ and $v_{y}=-1330 \mathrm{~km} / \mathrm{s}$ (median values), $7: v_{x}=340 \mathrm{~km} / \mathrm{s}$ and $v_{y}=-1190 \mathrm{~km} / \mathrm{s}, 8: v_{x}=340$ $\mathrm{km} / \mathrm{s}$ and $v_{y}=-1470 \mathrm{~km} / \mathrm{s}, 9: v_{x}=600 \mathrm{~km} / \mathrm{s}$ and $v_{y}=-1190 \mathrm{~km} / \mathrm{s}, 10: v_{x}=600 \mathrm{~km} / \mathrm{s}$ and $v_{y}=-1470$ $\mathrm{km} / \mathrm{s}$. All the orbits in both scenarios are bound. The time labels (filled squares) on the orbits are placed in intervals of 5 years, up to the year 2005 in the case of a BH and up to 2015 in the case of a neutrino ball.

Fig.4: Projected orbits of the star S0-1 in the case of a BH or neutrino ball with $M=2.4 \times 10^{6} M_{\odot}$. In this graph we explore how the orbits are affected by the uncertainty in the mass of the BH or neutrino ball. The orbits are calculated for $z=v_{z}=0$. The description of the orbits are the same as Fig. 3. The periods of S0-1 in the BH scenario vary between 11 and 20 years and all the orbits are bound in both scenarios.

Fig.5: Projected orbits of the star S0-1 for $M=2.8 \times 10^{6} M_{\odot}$. All the orbits are bound and calculated for different values of the velocity components as in Fig. 3. The periods of S0-1 vary between 9.5 years and 14.7 years in the $\mathrm{BH}$ scenario.

Fig. 6: The escape velocity as a function of the distance from Sgr $\mathrm{A}^{*}$ for $\mathrm{BH}$ and neutrino ball scenarios. The value of the mass of the central object is varied as indicated on the graph. The data points with error bars of 15 stars in the central $\operatorname{arcsec}^{2}$ are taken from Ghez et al. 1998 assuming that the projected velocity and distance from Sgr $\mathrm{A}^{*}$ are equal to the true velocity and distance, respectively, i.e. $z=0$ and $v_{z}=0$. This graph shows that $\mathrm{S} 0-1$ is bound in both scenarios for different values of the mass of the central object.

Fig. 7: The circular velocity as a function of the distance from $\mathrm{Sgr} \mathrm{A}^{*}$ for $\mathrm{BH}$ and neutrino ball scenarios. The mass of the central object is varied as indicated on the graph. The data points with error bars of 15 stars in the central $\operatorname{arcsec}^{2}$ are taken from Ghez et al. 1998 assuming that the projected velocity and distance from $\mathrm{Sgr} \mathrm{A}^{*}$ are equal to the true velocity and distance, respectively, i.e. $z=0$ and $v_{z}=0$.

This graph shows that the orbits of S0-1 are almost circular in the case of the BH scenario (see text for the discussion concerning the innermost data point). 
Fig. 8: Projected orbits of the star S0-1 in the case of a supermassive BH (top panel) and in the case of a neutrino ball (lower panel) with $M=2.6 \times 10^{6} M_{\odot}$. In this graph we explore how the orbits are affected by the uncertainty in the $z$-parameter. The labels for the orbits are given in the graph. Note, that for $z=0.4849^{\prime \prime}$, which corresponds to the radius of the neutrino ball for the assumed distance to the Galactic center, the orbits for a $\mathrm{BH}$ and neutrino ball are identical, as it should be. In this graph $v_{x}=470 \mathrm{~km} / \mathrm{s}$, $v_{y}=-1330 \mathrm{~km} / \mathrm{s}$ and $v_{z}=0$.

Fig.9: Projected orbits of the star S0-1 in the case of a supermassive BH (top panel) and in the case of a neutrino ball (lower panel) of $M=2.6 \times 10^{6} M_{\odot}$. In this graph we explore how the orbits are affected by the uncertainty in $v_{z}$. The labels for the orbits are given in the graph. Here, $v_{x}=470 \mathrm{~km} / \mathrm{s}, v_{y}=-1330$ $\mathrm{km} / \mathrm{s}$ and $z=0$.

Fig.10: Prediction regions for the supermassive central object. This graph combines line 9 from Fig. 4 and lines 2 and 4 from Fig. 5. If the star S0-1 will be found inside the ellipses (region $F$ ), this will rule out both the $\mathrm{BH}$ and the neutrino ball models. If the star S0-1 will eventually be found in the upper-left zone of the graph, i.e. up and left of the thick orbit, this will rule out the neutrino ball interpretation for the chosen neutrino mass. Finally, if S0-1 will be found to the right and below the thick line, then the supermassive central object should be interpreted either as a $\mathrm{BH}$ with large $z$ or as a neutrino ball. 


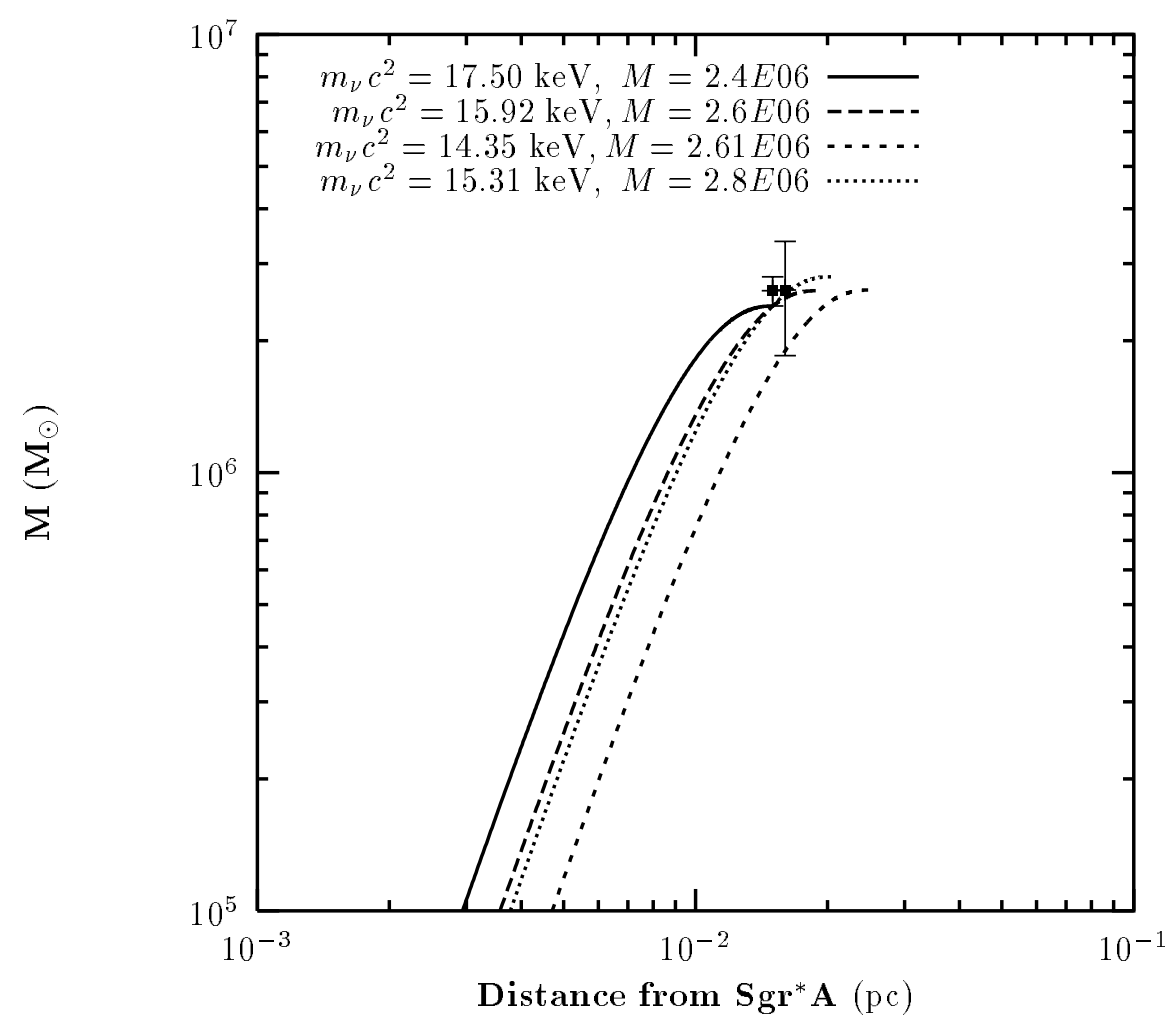

Fig. 1 


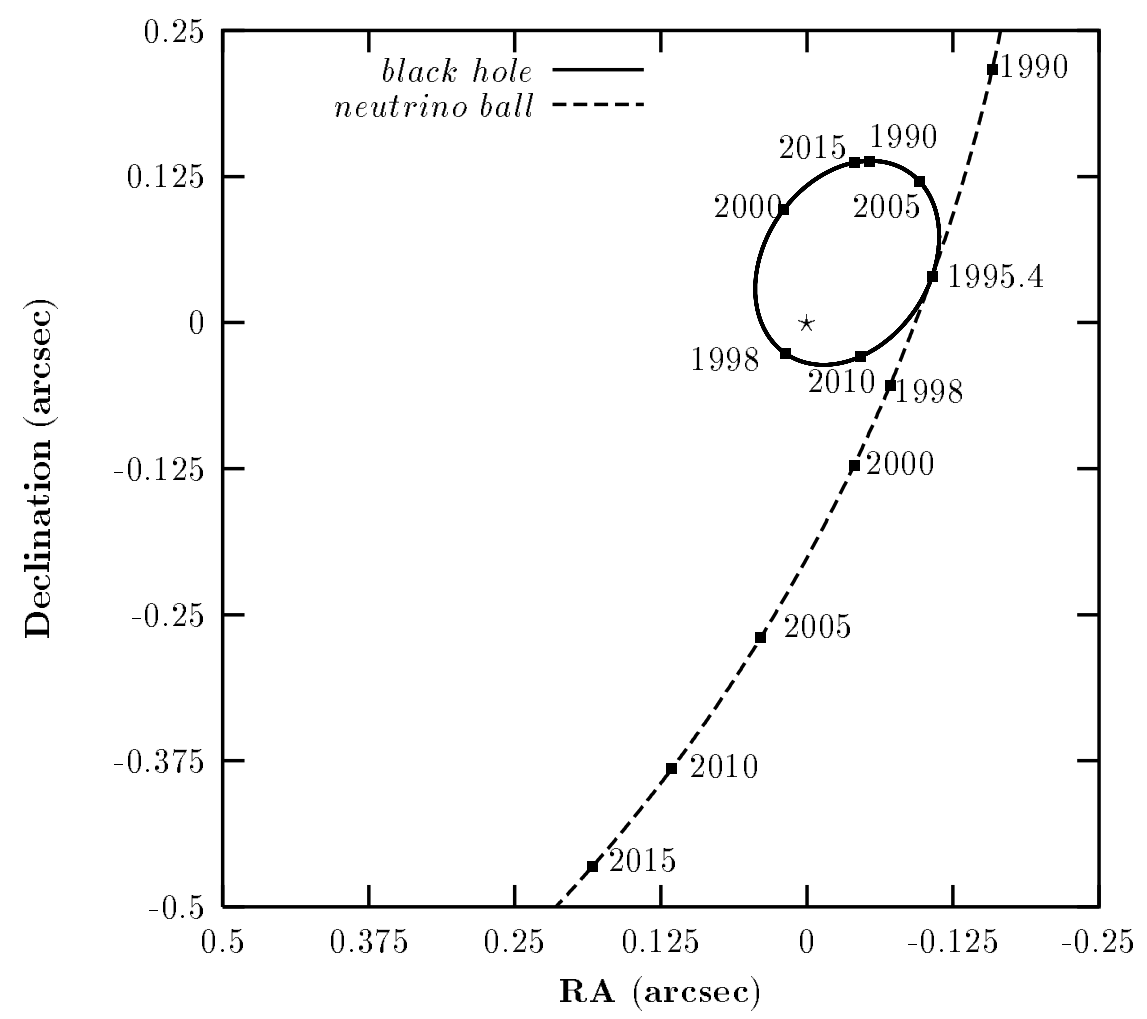

Fig. 2 


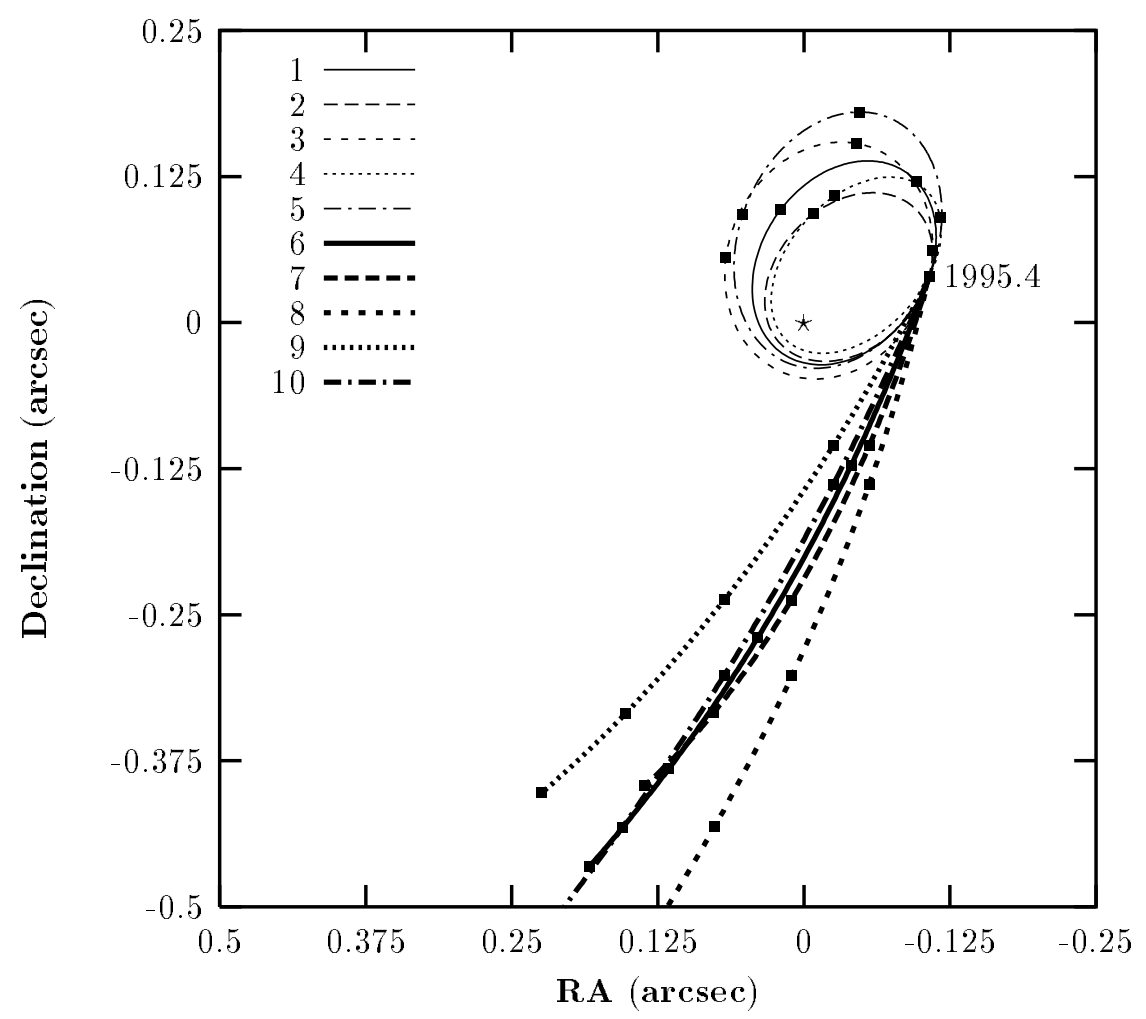

Fig. 3 


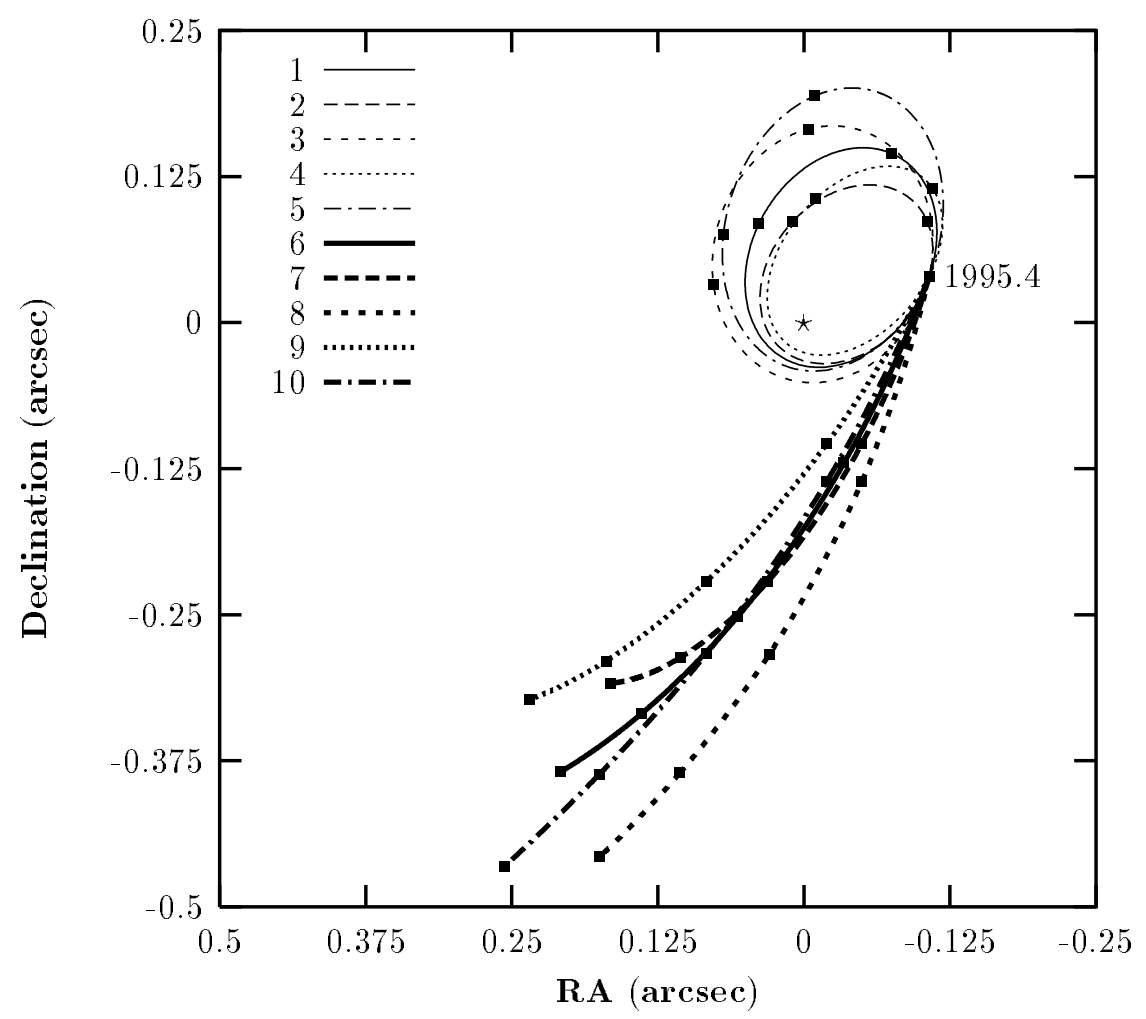

Fig. 4 


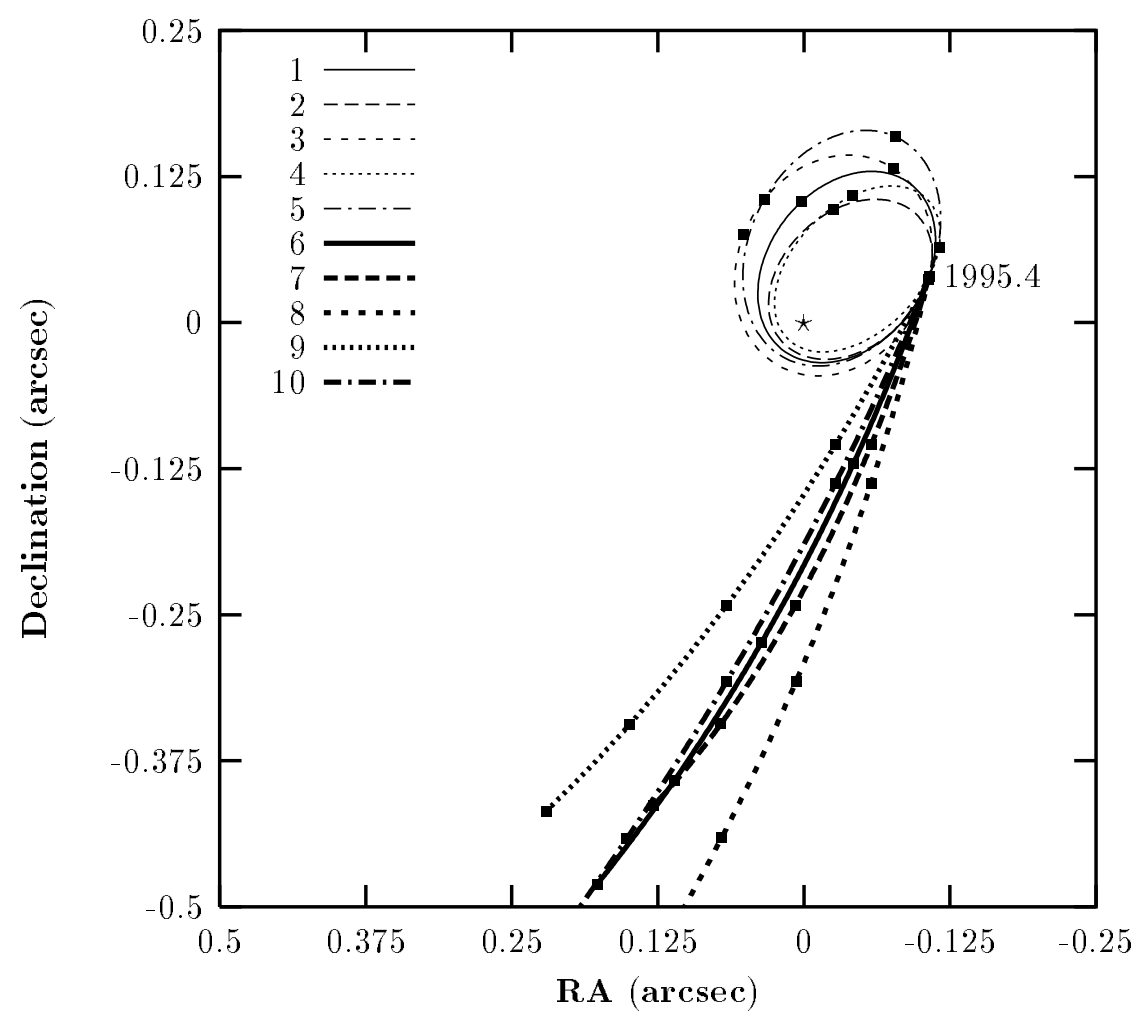

Fig. 5 


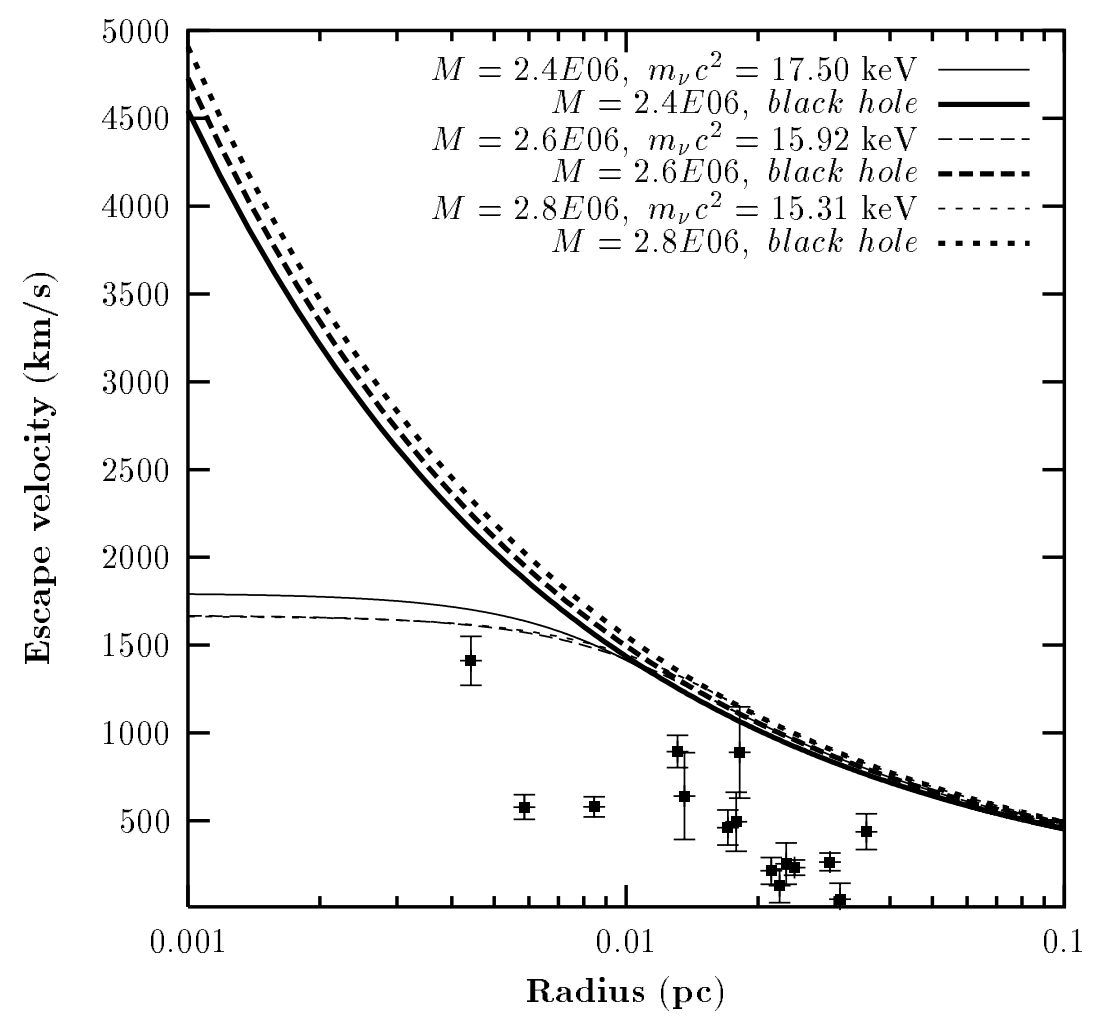

Fig. 6 


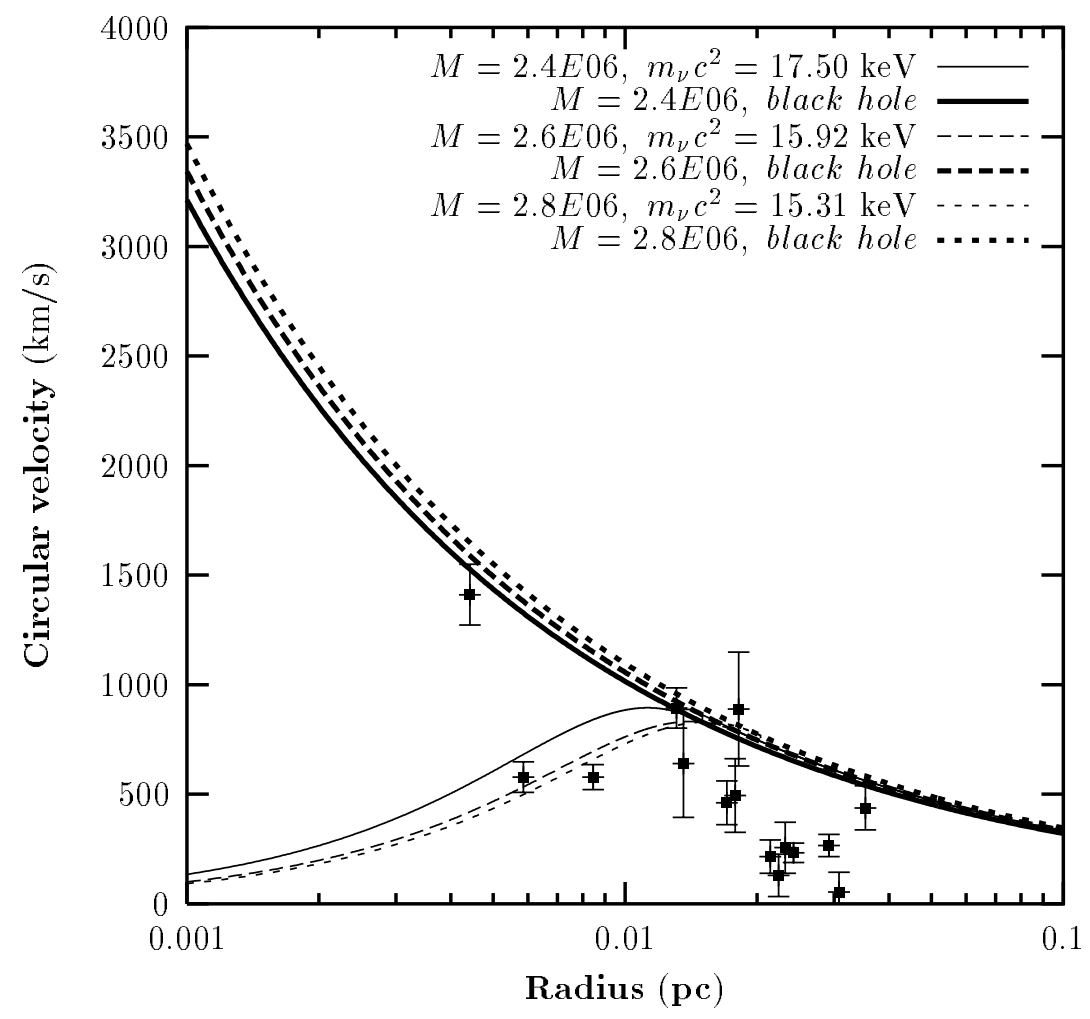

Fig. 7 
Fig. 8

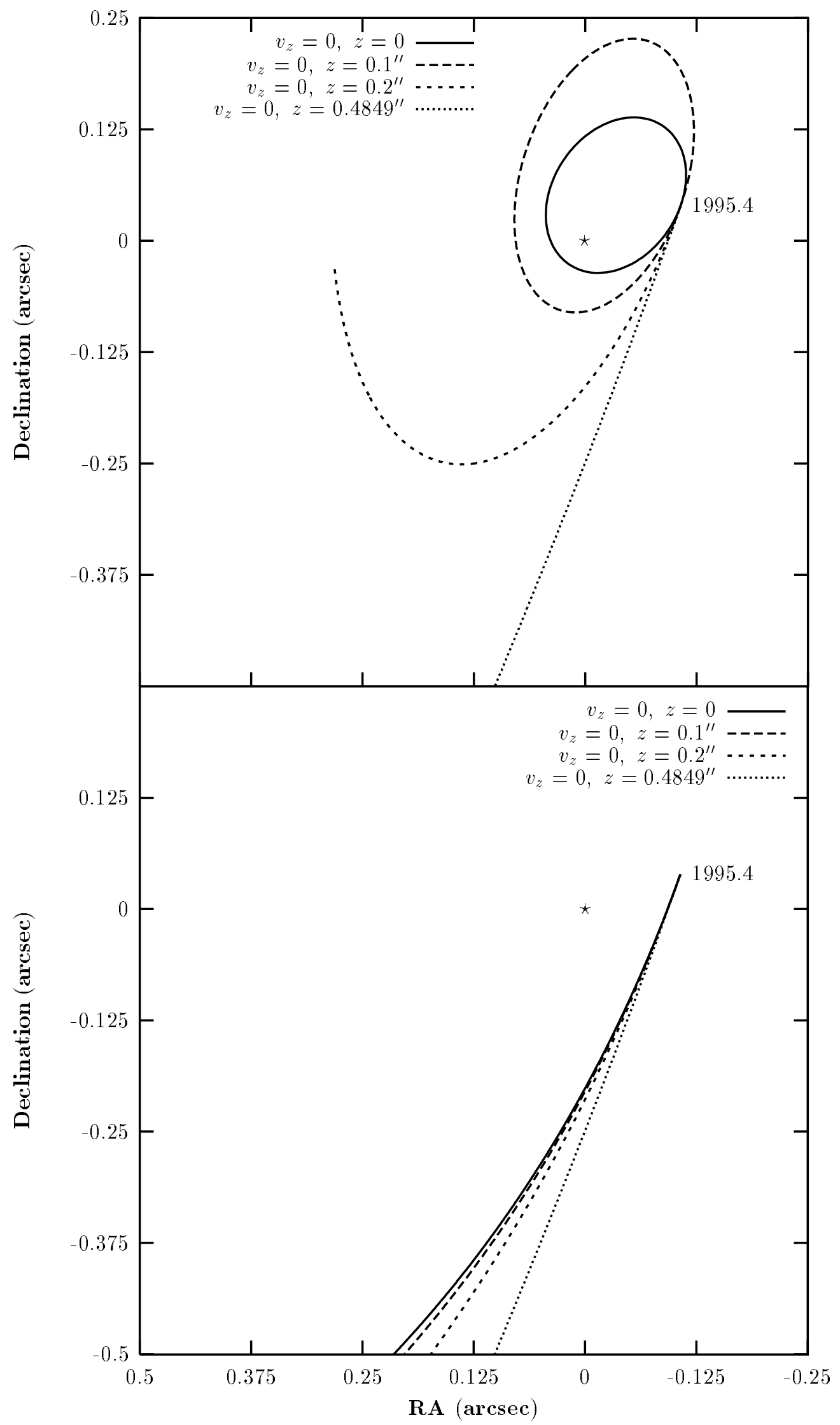


Fig.9

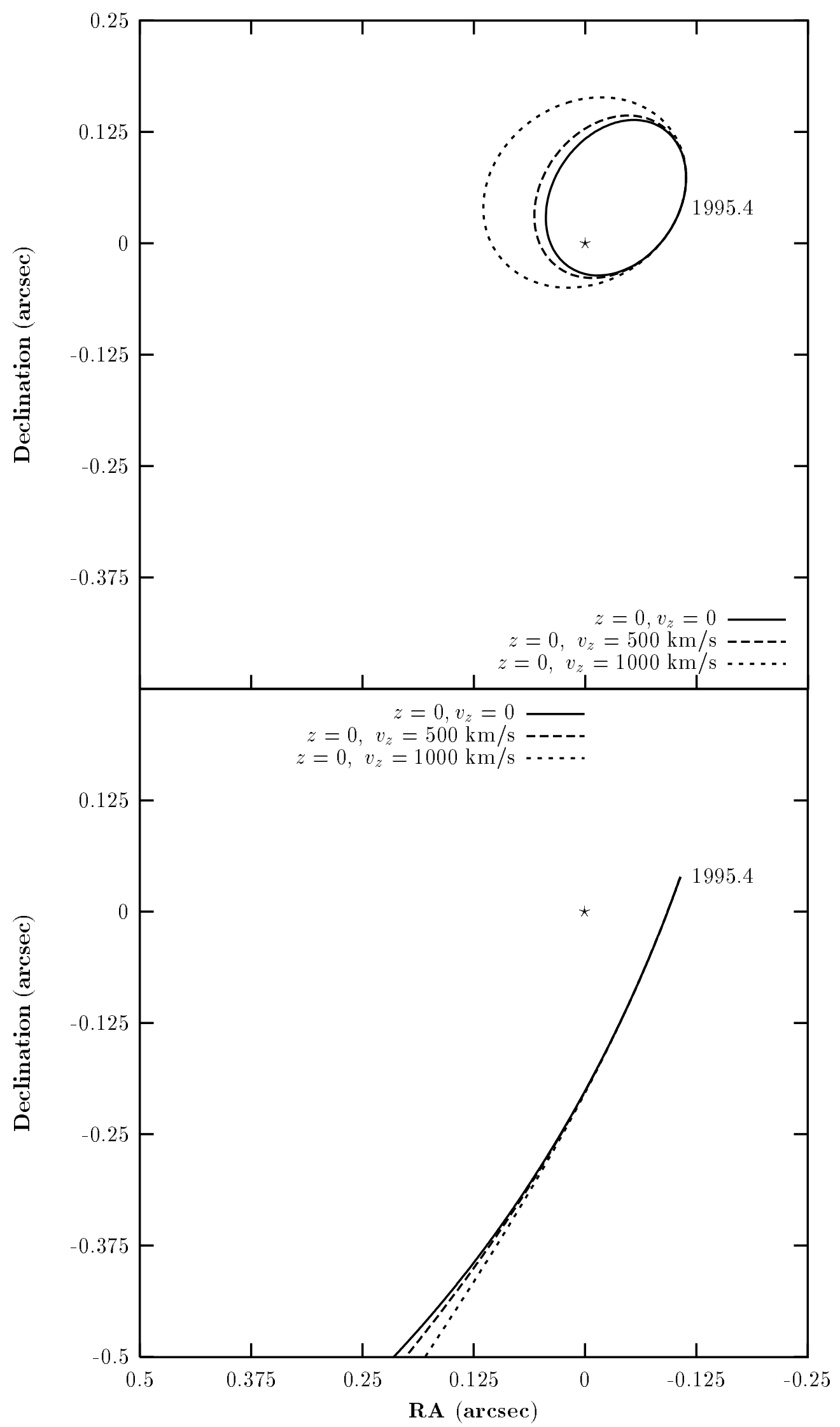




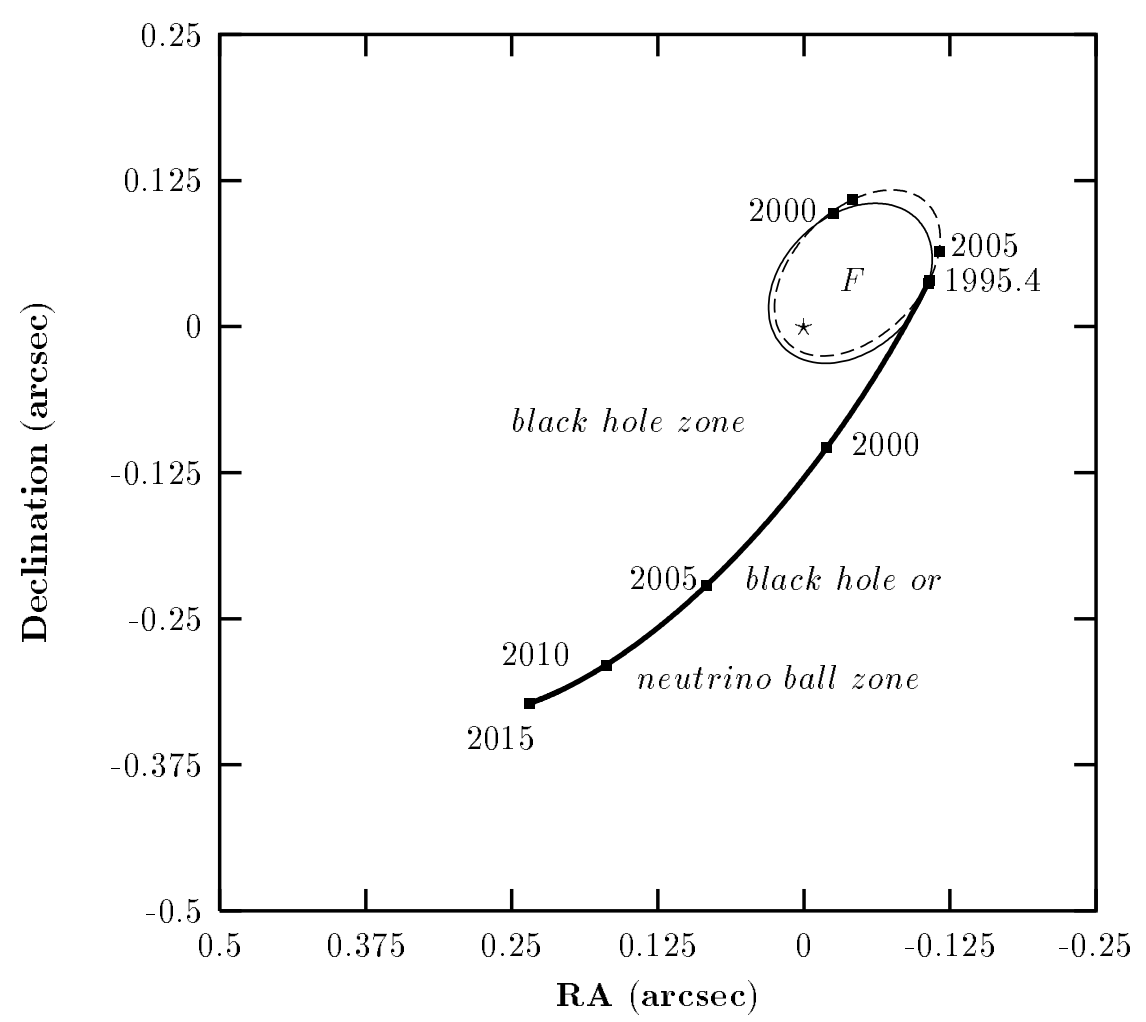

Fig. 10 